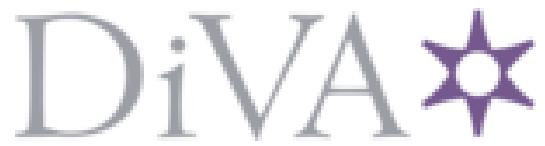

http://www.diva-portal.org

Postprint

This is the accepted version of a paper presented at 2021 IEEE Information Theory Workshop (ITW).

Citation for the original published paper:

Zhou, L., Oechtering, T J., Skoglund, M. (2021)

Polar Codes for Biometric Identification and Authentication

In: Kanazawa, Japan

https://doi.org/10.1109/ITW48936.2021.9611417

N.B. When citing this work, cite the original published paper.

Permanent link to this version:

http://urn.kb.se/resolve?urn=urn:nbn:se:kth:diva-306784 


\title{
Polar Codes for Biometric Identification and Authentication
}

\author{
Linghui Zhou, Student Member, IEEE, Tobias J. Oechtering, Senior Member, IEEE, \\ and Mikael Skoglund, Fellow, IEEE.
}

\begin{abstract}
In this work, we present a polar code design that offers a provably optimal solution for biometric identification systems allowing authentication under noisy enrollment with secrecy and privacy constraints. Binary symmetric memoryless source and channels are considered. It is shown that the proposed polar code design achieves the fundamental limits and satisfies more stringent secrecy constraints than previously in the literature. The proposed polar code design provides the first example of a code design that achieves the fundamental limits involving both identification and authentication.
\end{abstract}

\section{INTRODUCTION}

Biometrics is increasingly used in smart technology and devices. Different biometric features are used in various applications [1]. Two common application areas are biometric authentication and identification, which offer several advantages over traditional methods [2]. For example, biometric authentication and identification require the person to be present at the time of authentication and identification, which is a more stringent requirement and ensures a higher level of security. As the use of biometric data brings convenience, it also invokes privacy issues if the biometric information is compromised. Hence, the use of biometrics should be treated carefully.

Biometric systems for authentication and identification have been studied from various perspectives in the literature. The capacity of biometric identification systems is firstly characterized in [3]. Taking search complexity into consideration, hierarchical identification is studied in [4]-[7]. The fundamental limits of biometric systems considering privacy and secrecy aspects in various scenarios are studied in [7]-[13]. In [14], fundamental limits and relations of identification problems have been studied in general, including uncertainty models. Several fundamental limits have been also derived for biometric identification problems, which do provide insights on designing and analyzing biometric systems, but most of the analysis is based on the concept of typical sequences and cannot be implemented directly. Practical schemes of biometric identification that achieve fundamental limits, to the best of our knowledge, have not been studied before. In this work, we propose a polar code design that achieves the fundamental limits derived in [11] and satisfies strong secrecy, which is more stringent than weak secrecy in [11]. Lastly, though our code design achieves the optimal performance asymptotically,

Linghui Zhou, Tobias J. Oechtering, and Mikael Skoglund are with the Division of Information Science and Engineering, KTH Royal Institute of Technology, 11428 Stockholm, Sweden (e-mail: linghui@kth.se; oech@kth.se; skoglund@kth.se).

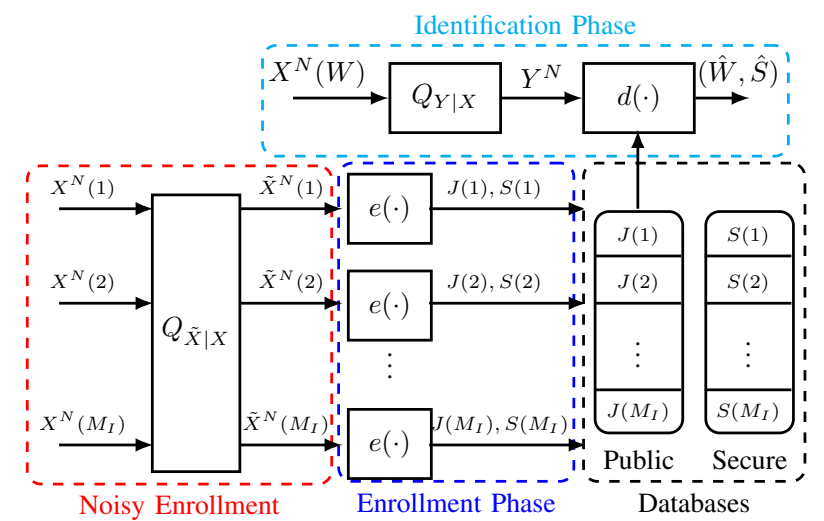

Fig. 1: Model of a biometric identification system that supports authentication under noisy enrollment.

they provides a framework for designing biometric systems and is promising with the development of more advanced techniques for constructing short block-length polar codes.

Notations: The set $\{1,2, \ldots, M\}$ is denoted with $[1: M]$. For $n \in \mathbb{N}^{+}$and $N \triangleq 2^{n}$, let $G_{N} \triangleq\left[\begin{array}{ll}1 & 0 \\ 1 & 1\end{array}\right]^{\otimes n} B_{N}$ denote the source polarization transformation defined in [15], where " $\otimes n$ ", denotes the $n$th Kronecker power and $B_{N}$ denotes the "bitreversal" permutation matrix. We denote the variational distance with $\mathbb{V}(\cdot, \cdot)$. Let $h_{2}(p)$ denote the binary entropy function $h_{2}(p)=-p \log _{2} p-(1-p) \log _{2}(1-p)$, where $p \in(0,1)$. Let $\oplus$ and $\ominus$ denote modulo addition and subtraction, which in our binary case are the XOR operation. Given a vector $V^{N}$ of length $N$, let $V^{N}[\mathcal{A}] \longleftarrow$ a denote assignment of vector a on coefficients of $V^{N}$ with indices in $\mathcal{A}$. For a set $\mathcal{A} \triangleq\left\{a_{j}\right\}_{j=1}^{|\mathcal{A}|}$, define $U^{N}[\mathcal{A}](w)=\left(U_{a_{1}}(w), U_{a_{2}}(w), \ldots, U_{a_{|\mathcal{A}|}}(w)\right)$. Given a random variable $\mathrm{A}$, let $|A|$ denote its length.

\section{Problem Formulation}

Consider a biometric identification system that supports authentication with noisy enrollment depicted in Fig. 1. Assume that there are $M_{I}$ users in the system with indices $\left\{1,2, \ldots, M_{I}\right\}$. We further assume that the biometric sequence $x^{N}(w)$ for each user $w \in\left[1: M_{I}\right]$ is identically independently distributed (i.i.d.) according to the probability mass function (p.m.f.) $Q_{X}(\cdot)$ on a finite alphabet $\mathcal{X}$. In the enrollment phase, for each user $w \in\left[1: M_{I}\right]$, the biometric sequence $x^{N}(w)$ is observed via a noisy discrete memoryless enrollment channel $Q_{\tilde{X} \mid X}$, which generates a noisy enrollment sequence $\tilde{x}^{N}(w)$. 
Then the enrollment mapping $e(\cdot)$ maps $\tilde{x}^{N}(w)$ to helper data $j(w) \in\left[1: M_{J}\right]$ and secret key $s(w) \in\left[1: M_{S}\right]$, i.e.,

$$
(j(w), s(w))=e\left(\tilde{x}^{N}(w)\right),
$$

where $j(w)$ and $s(w)$ are stored in a public helper database and a secure database at the location $w$, respectively.

In the identification phase, an unknown user $w$ with biometric source sequence $x^{N}(w)$ is observed via a discrete memoryless channel $Q_{Y \mid X}(\cdot)$ and a noisy observation $y^{N}$ is generated. The observed user's index $w$ is assumed to be a realization of a random variable $W$ that is uniformly distributed on $\left[1: M_{I}\right]$. The identification mapping $d(\cdot)$ uses $y^{N}$ and $\{j(i)\}_{i=1}^{M_{I}}$ to guess the user index denoted as $\hat{w}$ and estimate the secret key denoted as $\hat{s}$, i.e.,

$$
(\hat{w}, \hat{s})=d\left(y^{N},\{j(i)\}_{i=1}^{M_{I}}\right) .
$$

If the estimated secret key matches the secret key of guessed user $\hat{w}$, i.e., $\hat{s}=s(\hat{w})$, the user is granted access to the system.

The achievability of a rate tuple is defined as follows.

Definition 1: A rate tuple $\left(R_{I}, R_{S}, R_{L}, R_{J}\right) \in \mathbb{R}_{+}^{4}$ of the identification rate, the secret key rate, the privacy leakage rate, and the helper data rate is achievable in a biometric identification system allowing authentication under noisy enrollment if for all $\delta>0$ there exists some $N_{0}(\delta) \geq 1$ such that for all $N \geq N_{0}(\delta)$ there exists enrollment and identification mappings such that the following conditions are satisfied

$$
\begin{aligned}
& \operatorname{Pr}\{(\hat{W}, \hat{S}) \neq(W, S(W))\} \leq \delta, \\
& H(S(W))+\delta \geq \log M_{S} \geq N\left(R_{S}-\delta\right), \\
& \log M_{I} \geq N\left(R_{I}-\delta\right), \\
& I\left(S(W) ;\{J(i)\}_{i=1}^{M_{I}}\right) \leq \delta, \\
& I\left(X^{N}(W) ;\{J(i)\}_{i=1}^{M_{I}}\right) \leq N\left(R_{L}+\delta\right), \\
& \log M_{J} \leq N\left(R_{J}+\delta\right) .
\end{aligned}
$$

Moreover, we use $\mathcal{R}$ to denote the capacity region that contains all achievable rate tuples. Note that (6) ensures that strong secrecy holds, while [11] requires weak secrecy, i.e., $I\left(S(W) ;\{J(i)\}_{i=1}^{M_{I}}\right) \leq N \delta$. Since strong secrecy implies weak secrecy, by proving the achievability of strong secrecy, we have also shown that weak secrecy can be achieved.

Theorem 1 ( [11]): The capacity region of a biometric identification system that supports authentication under noisy enrollment is given by

$$
\begin{aligned}
\mathcal{R}=\{ & \left(R_{I}, R_{S}, R_{L}, R_{J}\right): R_{I}+R_{S} \leq I(U ; Y), \\
& R_{L} \geq I(U ; X)-I(U ; Y)+R_{I}, \\
& R_{J} \geq I(U ; \tilde{X})-I(U ; Y)+R_{I}, \\
& \text { for some } \left.P_{U X \tilde{X} Y}=Q_{X} Q_{\tilde{X} \mid X} Q_{Y \mid X} P_{U \mid \tilde{X}}\right\} .
\end{aligned}
$$

\section{Polar Code Design}

Codebook Generation: Let block length $N \triangleq 2^{n}$, where $n \in \mathbb{N}^{+}$. Introduce binary random variables $U$ and $V$. Fix an auxiliary p.m.f. $P_{U \mid \tilde{X}}$. Fix a sufficiently small $\epsilon>0$. Assume that there are $M_{I}=2^{N R_{I}}$ users. For each user $w \in[1$ : $\left.M_{I}\right]$, generate $u^{N}(w) \sim \prod_{i=1}^{N} P_{U \mid \tilde{X}}\left(u_{i}(w) \mid \tilde{x}_{i}(w)\right)$. Denote

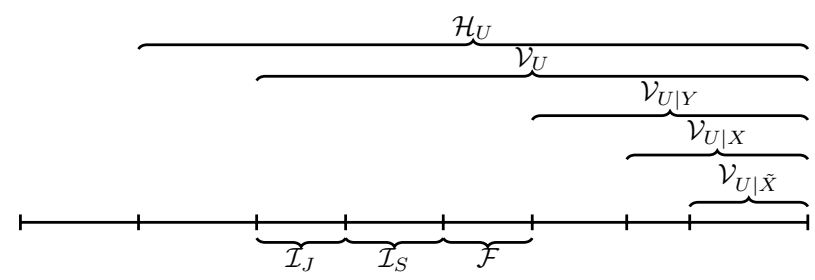

Fig. 2: An illustration of the subset structure of sets $\mathcal{V}_{U \mid \tilde{X}}$, $\mathcal{V}_{U \mid X}, \mathcal{V}_{U \mid Y}, \mathcal{V}_{U}, \mathcal{H}_{U}, \mathcal{F}, \mathcal{I}_{S}$, and $\mathcal{I}_{J}$. The line represents the set of indices, which are not ordered from 1 to $N$.

$v^{N}(w)=u^{N}(w) G_{N}$ the polar-code encoded codeword. For $\delta_{N} \triangleq 2^{-N^{\beta}}$ with an arbitrary but fixed $\beta \in(0,1 / 2)$, define the following sets on $[1: N]$ :

$$
\begin{aligned}
& \mathcal{H}_{U} \triangleq\left\{i: H\left(V^{i} \mid V^{i-1}\right) \geq \delta_{N}\right\}, \\
& \mathcal{V}_{U} \triangleq\left\{i: H\left(V^{i} \mid V^{i-1}\right) \geq 1-\delta_{N}\right\} \subset \mathcal{H}_{U}, \\
& \mathcal{V}_{U \mid Y} \triangleq\left\{i: H\left(V^{i} \mid V^{i-1}, Y^{N}\right) \geq 1-\delta_{N}\right\} \subset \mathcal{V}_{U}, \\
& \mathcal{V}_{U \mid X} \triangleq\left\{i: H\left(V^{i} \mid V^{i-1}, X^{N}\right) \geq 1-\delta_{N}\right\} \subset \mathcal{V}_{U \mid Y}, \\
& \mathcal{V}_{U \mid \tilde{X}} \triangleq\left\{i: H\left(V^{i} \mid V^{i-1}, \tilde{X}^{N}\right) \geq 1-\delta_{N}\right\} \subset \mathcal{V}_{U \mid X},
\end{aligned}
$$

where $Y^{N} \sim \prod_{i=1}^{N} Q_{Y \mid X}\left(y_{i} \mid x_{i}(w)\right)$ and the inclusion $\mathcal{V}_{U \mid \tilde{X}} \subset \mathcal{V}_{U \mid X} \subset \mathcal{V}_{U \mid Y}$ is due to $(U, V)-\tilde{X}-X-Y$. We pick a rate pair $\left(R_{I}, R_{S}\right)$ such that

$$
N\left(R_{I}+R_{S}+2 \epsilon\right)=\left|\mathcal{V}_{U} \backslash \mathcal{V}_{U \mid Y}\right| .
$$

We further define sets as follows.

Definition 2: Let $\mathcal{F}$ be a subset of $\mathcal{V}_{U} \backslash \mathcal{V}_{U \mid Y}$ such that

$$
\begin{aligned}
|\mathcal{F}| & =N \epsilon, \\
H\left(V^{i} \mid V^{i-1}, Y^{N}\right) & \geq H\left(V^{j} \mid V^{j-1}, Y^{N}\right),
\end{aligned}
$$

hold for all $i \in \mathcal{F}$ and any $j \in \mathcal{V}_{U} \backslash\left(\mathcal{V}_{U \mid Y} \cup \mathcal{F}\right)$. That is, $\mathcal{F}$ includes the indices in $\mathcal{V}_{U} \backslash \mathcal{V}_{U \mid Y}$ with the largest conditional entropy. Let $\mathcal{I}_{S}$ be any subset of $\mathcal{V}_{U} \backslash\left(\mathcal{V}_{U \mid Y} \cup \mathcal{F}\right)$ with size $N R_{S}$. Let $\mathcal{I}_{J}$ denote $\mathcal{V}_{U} \backslash\left(\mathcal{V}_{U \mid Y} \cup \mathcal{F} \cup \mathcal{I}_{S}\right)$ with size satisfying

$$
\left|\mathcal{I}_{J}\right|=\left|\mathcal{V}_{U} \backslash \mathcal{V}_{U \mid Y}\right|-N\left(\epsilon+R_{S}\right)=N\left(R_{I}+\epsilon\right) .
$$

The sets defined above have been illustrated in Fig. 2.

The above index sets of $v^{n}(w)$ can be interpreted as follows: (a) $\mathcal{H}_{U}$ is the index set of not sufficiently close to zero entropy given the previous bits; (b) $\mathcal{V}_{U}$ is the high entropy index set given the previous bits; (c) $\mathcal{V}_{U \mid Y}$ is the high entropy index set given the previous bits and $Y^{N}$; (d) $\mathcal{V}_{U \mid X}$ is the high entropy index set given the previous bits and $X^{N}$; (e) $\mathcal{V}_{U \mid \tilde{X}}$ is the high entropy index set given the previous bits and $\tilde{X}^{N}$; (f) $\mathcal{F}$ is a small set to ensure the source polar coding works; $(\mathrm{g})$ the bits in $v^{N}(w)$ related to indices in $\mathcal{I}_{J}$ and $\mathcal{I}_{S}$ are used to generate the helper data and the secret key, respectively.

Enrollment: The enrollment procedure is given in Algorithm 1. The system first constructs a source representation $\tilde{v}^{N}(w)$ of $\tilde{x}^{N}(w)$ for each user $w \in\left[1: M_{I}\right]$. The system uses part of the bits in the sequence $\tilde{v}^{N}(w)$ to generate the helper data and the secret key. 


$$
p_{\tilde{V}^{j}(w) \mid \tilde{V}^{j-1}(w), \tilde{X}^{N}{ }_{(w)}}\left(\tilde{v}^{j}(w) \mid \tilde{v}^{j-1}(w), \tilde{x}^{N}(w)\right)=\left\{\begin{array}{l}
p_{V^{j}(w) \mid V^{j-1}(w), \tilde{X}^{N}(w)}\left(\tilde{v}^{j}(w) \mid \tilde{v}^{j-1}(w), \tilde{x}^{N}(w)\right), \text { if } j \in \mathcal{H}_{U} \backslash \mathcal{V}_{U \mid \tilde{X}} \\
p_{V^{j}(w) \mid V^{j-1}(w)}\left(\tilde{v}^{j}(w) \mid \tilde{v}^{j-1}(w)\right), \text { if } j \in \mathcal{H}_{U}^{c}
\end{array}\right.
$$
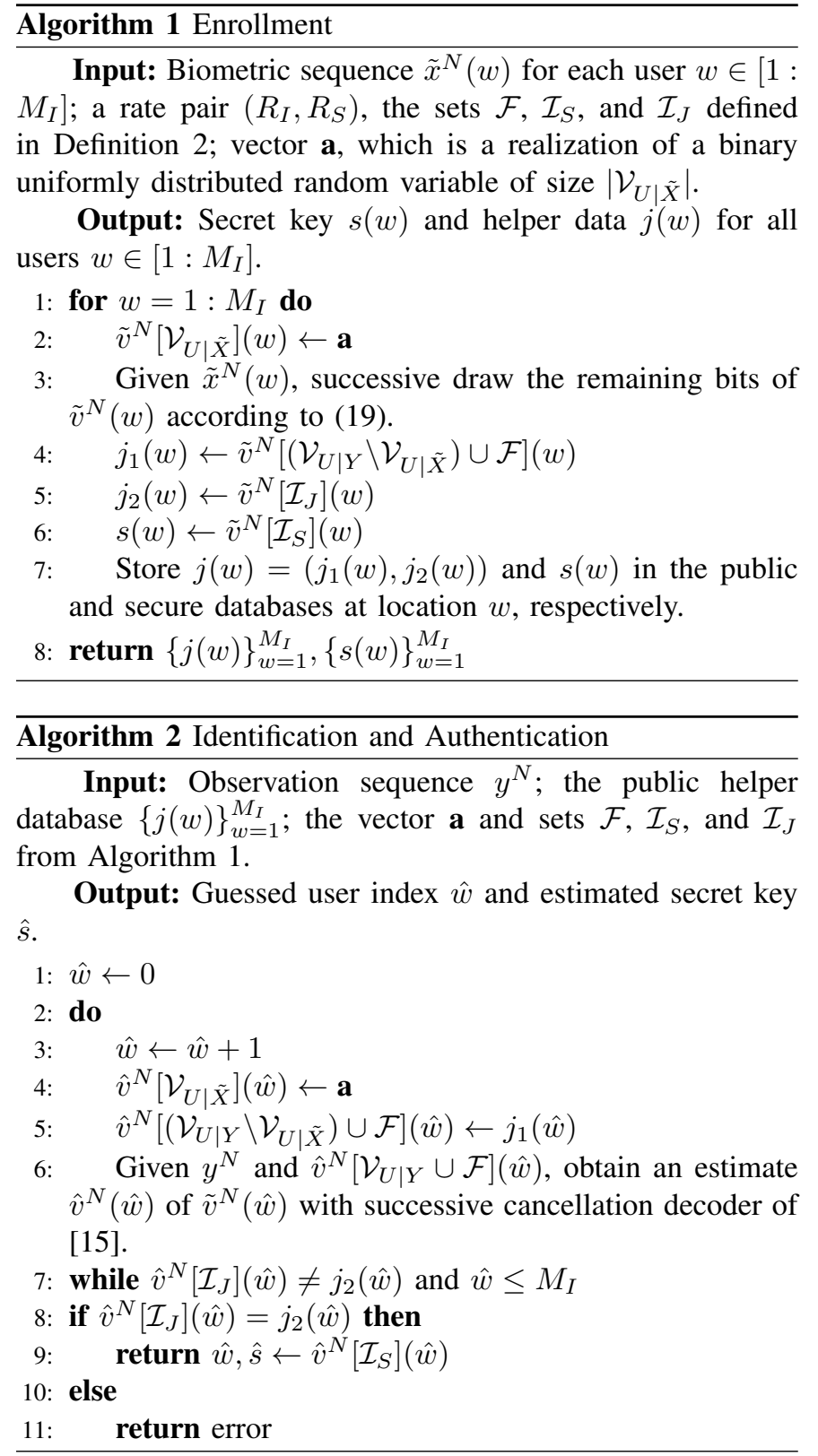

Identification and Authentication: The identification and authentication procedure is given in Algorithm 2. After observing $y^{N}$ generated by an unknown user, the system iteratively constructs an estimate $\hat{v}^{N}(\hat{w})$ of $\tilde{v}^{N}(\hat{w})$ for all $\hat{w} \in\left[1: M_{I}\right]$ using the first part $j_{1}(\hat{w})$ of the public helper data and the vector $A$ defined in Algorithm 1. Then the system compares $\hat{v}^{N}(\hat{w})$ with the second part $j_{2}(\hat{w})$ of the public helper data. If they match, i.e., $j_{2}(\hat{w})=\hat{v}^{N}\left[\mathcal{I}_{J}\right](\hat{w})$, the system outputs the guessed user index $\hat{w}$ and the estimated secret key $\hat{s}$. Otherwise, the system continues comparing with the next user when $\hat{w}<M_{I}$ or reports an error when $\hat{w}=M_{I}$.

The performance of the algorithms is ensured as follows.

Theorem 2: In a biometric identification system allowing authentication under noisy enrollment, for any rate tuple $\left(R_{I}, R_{S}, R_{L}, R_{J}\right) \in \mathcal{R}$, there exist a sequence of codes of length $2^{n}$ such that (3), (4), (5), (6), (7), and (8) are satisfied for $n \in \mathbb{N}^{+}$sufficiently large by the polar code design in Algorithm 1 and Algorithm 2.

\section{Proof of Theorem 2}

a) Rates Analysis: From (15), we have that

$$
\begin{aligned}
N\left(R_{I}+R_{S}\right) & =\left|\mathcal{V}_{U} \backslash \mathcal{V}_{U \mid Y}\right|-2 N \epsilon \\
& \stackrel{(a)}{=}\left|\mathcal{V}_{U}\right|-\left|\mathcal{V}_{U \mid Y}\right|-2 N \epsilon,
\end{aligned}
$$

where (a) holds because $\mathcal{V}_{U \mid Y} \subset \mathcal{V}_{U}$.

By [16, Lemma 1], we obtain that

$$
\lim _{N \rightarrow \infty} \frac{\left|\mathcal{V}_{U}\right|}{N}=H(U) \text {. }
$$

By [15, Theorem 1], we obtain that

$$
\begin{aligned}
& \lim _{N \rightarrow \infty} \frac{\left|\mathcal{V}_{U \mid \tilde{X}}\right|}{N}=H(U \mid \tilde{X}), \\
& \lim _{N \rightarrow \infty} \frac{\left|\mathcal{V}_{U \mid Y}\right|}{N}=H(U \mid Y), \\
& \lim _{N \rightarrow \infty} \frac{\left|\mathcal{V}_{U \mid X}\right|}{N}=H(U \mid X) .
\end{aligned}
$$

Combining (20), (21), and (23), we have that

$$
\lim _{N \rightarrow \infty} R_{I}+R_{S}=I(U ; Y)-2 \epsilon .
$$

The helper data rate $R_{J}$ is

$$
\begin{aligned}
R_{J} & =\frac{|J(W)|}{N}=\frac{\left|\mathcal{V}_{U \mid Y} \backslash \mathcal{V}_{U \mid \tilde{X}}\right|+|\mathcal{F}|+\left|\mathcal{I}_{J}\right|}{N} \\
& \stackrel{(a)}{=} \frac{\left|\mathcal{V}_{U \mid Y}\right|-\left|\mathcal{V}_{U \mid \tilde{X}}\right|+N\left(R_{I}+2 \epsilon\right)}{N},
\end{aligned}
$$

where (a) follows from $\mathcal{V}_{U \mid \tilde{X}} \subset \mathcal{V}_{U \mid Y}$ and (18).

Combining (23), (26), and (22), we have that

$$
\lim _{N \rightarrow \infty} R_{J}=I(U ; \tilde{X})-I(U ; Y)+R_{I}+2 \epsilon .
$$

b) Error Events Analysis: Assume that user $W$ is observed. Let $J(W)=\left(J_{1}(W), J_{2}(W)\right)$ and $S(W)$ denote the actual helper data and the secret key of user $W$. Define the following error events

$$
\begin{aligned}
& \mathcal{E}_{1}=\left\{\hat{V}^{N}\left[\mathcal{I}_{J}\right](W) \neq\right.\left.J_{2}(W)\right\}, \\
& \mathcal{E}_{2}=\left\{\exists \hat{w} \neq W: \hat{V}^{N}\left[\mathcal{I}_{J}\right](\hat{w})=J_{2}(\hat{w}),\right. \\
&\left.\hat{V}^{N}\left[\mathcal{I}_{S}\right](\hat{w})=S(W)\right\}, \\
& \mathcal{E}_{3}=\left\{\hat{V}^{N}\left[\mathcal{I}_{J}\right](W)=J_{2}(W), \hat{V}^{N}\left[\mathcal{I}_{S}\right](W) \neq S(W)\right\},
\end{aligned}
$$




$$
\begin{array}{r}
\mathcal{E}_{4}=\left\{\exists \hat{w} \neq W: \hat{V}^{N}\left[\mathcal{I}_{J}\right](\hat{w})=J_{2}(\hat{w}),\right. \\
\left.\hat{V}^{N}\left[\mathcal{I}_{S}\right](\hat{w}) \neq S(W)\right\} .
\end{array}
$$

The first two events are identification error events that the correct user is not identified: $\mathcal{E}_{1}$ denotes the case that the true user does not satisfy the equality condition in the comparison procedure; $\mathcal{E}_{2}$ denotes the case that there exists another user that satisfies the equality condition and the estimated secret key matches the true one. The third and fourth error events are the authentication errors that the estimated secret key does not match the true one: $\mathcal{E}_{3}$ denotes the case that the guessed user $\hat{w}$ is the same as the true one but the estimated secret key does not match the true secret key $S(W)$; $\mathcal{E}_{4}$ denotes the case that neither the guessed user index nor the estimated secret key is the same as the true helper data index and the secret key of the observed user. The identification and authentication are reliable if and only if none of the above events happen. We define the error event

$$
\mathcal{E}=\{(\hat{W}, \hat{S}) \neq(W, S(W))\}
$$

and it holds that

$$
\mathcal{E} \subset \mathcal{E}_{1} \cup \mathcal{E}_{2} \cup \mathcal{E}_{3} \cup \mathcal{E}_{4}
$$

Before proceeding with bounding the probability of $\mathcal{E}$, we include the following lemma. Let $a_{1}, a_{2}, \ldots, a_{\left|\mathcal{I}_{J}\right|}$ denote the elements of the set $\mathcal{I}_{J}$ such that $a_{1}<a_{2}<\ldots<a_{\left|\mathcal{I}_{J}\right|}$. Let $\mathcal{I}_{J}^{i}=\left\{a_{1}, a_{2}, \ldots, a_{i}\right\}$.

Lemma 1: Assume that user $w$ is observed, then it holds that

$$
\operatorname{Pr}\left\{\hat{V}^{N}(w) \neq \tilde{V}^{N}(w)\right\} \stackrel{N \rightarrow \infty}{\longrightarrow} 0 .
$$

Proof: In the identification and authentication algorithms, $\hat{V}\left[\mathcal{I}_{J} \cup \mathcal{I}_{S}\right](w)$ is obtained by successive cancellation operation with $Y^{N}$ and $\tilde{V}\left[\mathcal{V}_{U \mid Y} \cup \mathcal{F}\right](w)$. Next we consider the size of $\mathcal{V}_{U \mid Y} \cup \mathcal{F}$.

From (23), we have that for sufficiently small $\epsilon>0$ there exists $N_{0}(\epsilon) \in \mathbb{N}^{+}$such that for any $N=2^{n} \geq N_{0}(\epsilon)$ for some $n \in \mathbb{N}^{+}$, it holds that $\left|\mathcal{V}_{U \mid Y}\right| / N>H(U \mid Y)-\epsilon$. Therefore, for sufficiently large $N$, it holds that

$$
\left|\mathcal{V}_{U \mid Y} \cup \mathcal{F}\right|=\left|\mathcal{V}_{U \mid Y}\right|+|\mathcal{F}|>N H(U \mid Y) .
$$

Applying [15, Theorem 3], we can obtain (31) from (32).

Lemma 2: For any $w \in\left[1: M_{I}\right]$ and $i \in\left[2:\left|\mathcal{I}_{J}\right|\right]$, it holds that

$$
H\left(\tilde{V}_{a_{i}}(w) \mid \tilde{V}^{a_{i-1}}(w)\right) \stackrel{N \rightarrow \infty}{\longrightarrow} 1 .
$$

Proof: See [17, Appendix B-C].

In the following, due to symmetry and without loss of generality, assume that $W=1$. Now we consider the probability of $\mathcal{E}_{1} \cup \mathcal{E}_{3} \mid W=1$ as follows

$$
\begin{aligned}
& \operatorname{Pr}\left\{\mathcal{E}_{1} \cup \mathcal{E}_{3} \mid W=1\right\} \\
& =\operatorname{Pr}\left\{\left(\hat{V}^{N}\left[\mathcal{I}_{J}\right](1), \hat{V}^{N}\left[\mathcal{I}_{S}\right](1)\right) \neq\left(J_{2}(1), S(1)\right) \mid W=1\right\} \\
& =\operatorname{Pr}\left\{\hat{V}^{N}\left[\mathcal{I}_{J} \cup \mathcal{I}_{S}\right](1) \neq \tilde{V}^{N}\left[\mathcal{I}_{J} \cup \mathcal{I}_{S}\right](1) \mid W=1\right\} \\
& \stackrel{N \rightarrow \infty}{\longrightarrow} 0
\end{aligned}
$$

where the last step follows from Lemma 1.

The probability of $\mathcal{E}_{2} \cup \mathcal{E}_{4} \mid W=1$ can be bounded as follows

$$
\begin{aligned}
\operatorname{Pr} & \left\{\mathcal{E}_{2} \cup \mathcal{E}_{4} \mid W=1\right\} \\
= & \operatorname{Pr}\left\{\exists \hat{w} \neq 1: \hat{V}^{N}\left[\mathcal{I}_{J}\right](\hat{w})=J_{2}(\hat{w}) \mid W=1\right\} \\
\leq & \sum_{\hat{w} \neq 1} \operatorname{Pr}\left\{\hat{V}^{N}\left[\mathcal{I}_{J}\right](\hat{w})=J_{2}(\hat{w}) \mid W=1\right\} \\
= & \sum_{\hat{w} \neq 1} \operatorname{Pr}\left\{\forall i \in \mathcal{I}_{J}: \hat{V}_{i}(\hat{w})=\tilde{V}_{i}(\hat{w}) \mid W=1\right\} \\
= & \sum_{\hat{w} \neq 1} \prod_{i=1}^{\left|\mathcal{I}_{J}\right|} \operatorname{Pr}\left\{\hat{V}_{a_{i}}(\hat{w})=\tilde{V}_{a_{i}}(\hat{w}) \mid W=1,\right. \\
& \left.\hat{V}^{a_{i-1}}\left[\mathcal{I}_{J}^{i-1}\right](\hat{w})=\tilde{V}^{a_{i-1}}\left[\mathcal{I}_{J}^{i-1}\right](\hat{w})\right\} .
\end{aligned}
$$

To bound the probability above, we firstly consider the following conditional entropy. For $\hat{w} \neq 1$, it holds that

$$
\begin{aligned}
& H\left(\hat{V}_{a_{i}}(\hat{w}) \oplus \tilde{V}_{a_{i}}(\hat{w}) \mid\right. \\
& \left.\quad \hat{V}^{a_{i-1}}\left[\mathcal{I}_{J}^{i-1}\right](\hat{w})=\tilde{V}^{a_{i-1}}\left[\mathcal{I}_{J}^{i-1}\right](\hat{w}), W=1\right) \\
& \stackrel{(a)}{\geq} H\left(\hat{V}_{a_{i}}(\hat{w}) \oplus \tilde{V}_{a_{i}}(\hat{w}) \mid \hat{V}^{a_{i-1}}(\hat{w})=\tilde{V}^{a_{i-1}}(\hat{w}), W=1\right) \\
& \stackrel{(a)}{\geq} H\left(\hat{V}_{a_{i}}(\hat{w}) \oplus \tilde{V}_{a_{i}}(\hat{w}) \mid \hat{V}^{a_{i}}(\hat{w}), \tilde{V}^{a_{i-1}}(\hat{w}), Y^{N}, W=1\right) \\
& =H\left(\tilde{V}_{a_{i}}(\hat{w}) \mid \hat{V}^{a_{i}}(\hat{w}), \tilde{V}^{a_{i-1}}(\hat{w}), Y^{N}, W=1\right) \\
& \stackrel{(b)}{=} H\left(\tilde{V}_{a_{i}}(\hat{w}) \mid \tilde{V}^{a_{i-1}}(\hat{w}), Y^{N}, W=1\right) \\
& \stackrel{(c)}{=} H\left(\tilde{V}_{a_{i}}(\hat{w}) \mid \tilde{V}^{a_{i-1}}(\hat{w})\right) \\
& \stackrel{(d)}{\rightarrow} 1,
\end{aligned}
$$

where (a) holds since conditioning reduces entropy; (b) holds because $\hat{V}^{a_{i}}(\hat{w})$ is a function of $\left(\tilde{V}^{a_{i-1}}(\hat{w}), Y^{N}\right)$ due to the successive cancellation operation; (c) holds because $Y^{N}$ is the observation of user $W$ and thus is independent of $\left(X^{N}(\hat{w}), \tilde{V}^{N}(\hat{w})\right)$ for $\hat{w} \neq 1$; (d) follows from Lemma 2 .

Since $\hat{V}_{a_{i}}(\hat{w}) \oplus \tilde{V}_{a_{i}}(\hat{w})$ is binary, (35) implies that for any $\epsilon^{\prime}>0$ and sufficiently large $N$, we have

$$
\begin{aligned}
\operatorname{Pr}\left\{\hat{V}_{a_{i}}(\hat{w}) \oplus \tilde{V}_{a_{i}}(\hat{w})\right. & =0 \mid W=1, \\
\hat{V}^{a_{i-1}}\left[\mathcal{I}_{J}^{i-1}\right](\hat{w}) & \left.=\tilde{V}^{a_{i-1}}\left[\mathcal{I}_{J}^{i-1}\right](\hat{w})\right\}<\frac{1}{2}+\epsilon^{\prime} .
\end{aligned}
$$

Now we consider $\epsilon^{\prime} \in\left(0, \frac{2^{\frac{\epsilon}{R_{I}+\epsilon}}-1}{2}\right)$ for the fixed $\epsilon>0$. Combining (34) and (36), we obtain that

$$
\begin{aligned}
\operatorname{Pr} & \left\{\mathcal{E}_{2} \cup \mathcal{E}_{4} \mid W=1\right\} \leq \sum_{\hat{w} \neq 1} \prod_{i=1}^{\left|\mathcal{I}_{J}\right|}\left(\frac{1}{2}+\epsilon^{\prime}\right)=2^{N R_{I}}\left(\frac{1}{2}+\epsilon^{\prime}\right)^{\left|\mathcal{I}_{J}\right|} \\
& \stackrel{(a)}{=} 2^{N R_{I}}\left(\frac{1}{2}+\epsilon^{\prime}\right)^{N\left(R_{I}+\epsilon\right)}=\frac{\left(1+2 \epsilon^{\prime}\right)^{N\left(R_{I}+\epsilon\right)}}{2^{N \epsilon}} \\
& \leq\left(\frac{\left(1+2 \epsilon^{\prime}\right)^{\left(R_{I}+\epsilon\right)}}{2^{\epsilon}}\right)^{N} \stackrel{(b)}{\longrightarrow} 0
\end{aligned}
$$

as $N \rightarrow \infty$; where (a) follows from (18); (b) follows from $\frac{\left(1+2 \epsilon^{\prime}\right)^{\left(R_{I}+\epsilon\right)}}{2^{\epsilon}}<1$ since $\epsilon^{\prime}<\frac{2^{R_{I}+\epsilon}-1}{2}$.

Combining (33) and (37), we can conclude that

$$
\operatorname{Pr}\{\mathcal{E}\} \rightarrow 0,
$$


as $N \rightarrow \infty$. Therefore, we can conclude that there exists a suitable codebook $\mathcal{C}=C$ such that (38) holds.

c) Uniformity of Secret Keys: We firstly include the following lemmas to show the uniformity of secret keys. Let $\mathcal{D}$ denote $\mathcal{V}_{U} \backslash \mathcal{V}_{U \mid \tilde{X}}$ for simplicity.

Lemma 3: Let 1-dim distribution $q_{\mathcal{U}_{|\mathcal{D}|}}$ denote a uniform distribution over $\left[1: 2^{|\mathcal{D}|}\right]$. For any $w \in\left[1: M_{I}\right]$, it holds that

$$
\mathbb{V}\left(p_{\left.\tilde{V}^{N}[\mathcal{D}](w)\right)}, q_{\mathcal{U}_{|\mathcal{D}|}}\right) \leq 2 \sqrt{2 \ln 2} \sqrt{N \delta_{N}}
$$

Proof: See [17, Appendix B-D].

Lemma 4: For any $w \in\left[1: M_{I}\right]$, it holds that

$$
|\mathcal{D}|-H\left(\tilde{V}^{N}[\mathcal{D}](w)\right) \leq \delta
$$

where $\delta \rightarrow 0$ as $N \rightarrow \infty$. Consequently, it holds that

$$
\begin{aligned}
& \left|\mathcal{I}_{S}\right|-H\left(\tilde{V}^{N}\left[\mathcal{I}_{S}\right](w)\right) \leq \delta, \\
& I\left(\tilde{V}^{N}\left[\left(\mathcal{V}_{U \mid Y} \backslash \mathcal{V}_{U \mid \tilde{X}}\right) \cup \mathcal{I}_{J}\right](w) ; \tilde{V}^{N}\left[\mathcal{I}_{S}\right](w)\right) \leq \delta .
\end{aligned}
$$

Proof: Similar to [16, Appendix B-C], we that have

$$
\begin{aligned}
& |\mathcal{D}|-H\left(\tilde{V}^{N}[\mathcal{D}](w)\right) \\
& \stackrel{(a)}{\leq} \mathbb{V}\left(p_{\tilde{V}^{N}[\mathcal{D}](w)}, q_{\mathcal{U}_{|\mathcal{D}|}}\right) \times \log _{2} \frac{|\mathcal{D}|}{\mathbb{V}\left(p_{\tilde{V}^{N}[\mathcal{D}](w)}, q_{\mathcal{U}_{|\mathcal{D}|}}\right)} \\
& \stackrel{(b)}{\leq} N \mathbb{V}\left(p_{\tilde{V}^{N}[\mathcal{D}](w)}, q_{\mathcal{U}_{|\mathcal{D}|}}\right) \\
& \quad-\mathbb{V}\left(p_{\tilde{V}^{N}[\mathcal{D}](w)}, q_{\mathcal{U}_{|\mathcal{D}|}}\right) \times \log _{2} \mathbb{V}\left(p_{\tilde{V}^{N}[\mathcal{D}](w)}, q_{\mathcal{U}_{|\mathcal{D}|}}\right) \\
& \stackrel{(c)}{\leq} 2 \sqrt{2 \ln 2} \sqrt{N \delta_{N}}\left(N-\log _{2}\left(2 \sqrt{2 \ln 2} \sqrt{N \delta_{N}}\right)\right) \\
& \stackrel{(d)}{\rightarrow} 0,
\end{aligned}
$$

as $N \rightarrow \infty$; where (a) follows from [18, Lemma 2.7]; (b) follows from $\log |\mathcal{D}| \leq N$; (c) holds due to Lemma 3 and the fact that $x \log _{2} x$ is decreasing for sufficiently small $x>0$; (d) follows from the fact that $N \sqrt{N \delta_{N}} \rightarrow 0$ and $N \delta_{N} \rightarrow 0$ as $N \rightarrow \infty$. This completes the proof of Lemma 4 .

The term $|\mathcal{D}|-H\left(\tilde{V}^{N}[\mathcal{D}](w)\right)$ can be rewritten as follows

$$
\begin{aligned}
|\mathcal{D}| & -H\left(\tilde{V}^{N}[\mathcal{D}](w)\right) \\
= & \left|\left(\mathcal{V}_{U \mid Y} \backslash \mathcal{V}_{U \mid \tilde{X}}\right) \cup \mathcal{I}_{J}\right|-H\left(\tilde{V}^{N}\left[\left(\mathcal{V}_{U \mid Y} \backslash \mathcal{V}_{U \mid \tilde{X}}\right) \cup \mathcal{I}_{J}\right](w)\right) \\
& +\left|\mathcal{I}_{S}\right|-H\left(\tilde{V}^{N}\left[\mathcal{I}_{S}\right](w)\right) \\
& +I\left(\tilde{V}^{N}\left[\left(\mathcal{V}_{U \mid Y} \backslash \mathcal{V}_{U \mid \tilde{X}}\right) \cup \mathcal{I}_{J}\right](w) ; \tilde{V}^{N}\left[\mathcal{I}_{S}\right](w)\right)
\end{aligned}
$$

Since the entropy of a random variable is no larger than its cardinality, combining (43) and (44), we obtain that (41) and (42) hold. This completes the proof of Lemma 4.

From (41), we obtain that

$$
\begin{gathered}
H(S(W) \mid \mathcal{C}=C)=\sum_{w=1}^{M_{I}} \operatorname{Pr}(W=w) H(S(w) \mid \mathcal{C}=C) \\
\geq \sum_{w=1}^{M_{I}} \operatorname{Pr}(W=w)|S(w)|-\delta=|S(W)|-\delta
\end{gathered}
$$

where $\delta \rightarrow 0$ as $N \rightarrow \infty$. d) Secrecy Analysis: The secrecy leakage can be bounded as

$$
\begin{aligned}
& \left.I\left(S(W) ;\{J(i)\}_{i=1}^{M_{I}}\right) \mid \mathcal{C}=C\right) \\
& \stackrel{(a)}{=} I(S(W) ; J(W) \mid \mathcal{C}=C) \\
& =\sum_{w=1}^{M_{I}} \operatorname{Pr}(W=w) I(S(w) ; J(w) \mid \mathcal{C}=C) \\
& =\sum_{w=1}^{M_{I}} \frac{I\left(\tilde{V}^{N}\left[\mathcal{I}_{S}\right](w) ; \tilde{V}^{N}\left[\left(\mathcal{V}_{U \mid Y} \backslash \mathcal{V}_{U \mid \tilde{X}}\right) \cup \mathcal{I}_{J}\right](w) \mid \mathcal{C}=C\right)}{M_{I}} \\
& \stackrel{(b)}{\leq} \delta,
\end{aligned}
$$

where $\delta \rightarrow 0$ as $N \rightarrow \infty$; (a) follows from the fact that the secret key of user $W$ is independent of the helper data of the other users; (b) follows from (42) in Lemma 4.

e) Privacy Analysis: To bound the privacy leakage, we include the following lemmas.

Lemma 5: It holds that

$$
I\left(\tilde{V}^{N}\left[\mathcal{V}_{U \mid X}\right](W) ; X^{N}(W) \mid \mathcal{C}=C\right) \leq \delta
$$

where $\delta \rightarrow 0$ as $N \rightarrow \infty$.

Proof: See [17, Appendix B-G].

Now we consider the privacy leakage as follows

$$
\begin{aligned}
& I\left(\{J(i)\}_{i=1}^{M_{I}} ; X^{N}(W) \mid \mathcal{C}=C\right) \\
& \stackrel{(a)}{=} I\left(J(W) ; X^{N}(W) \mid \mathcal{C}=C\right) \\
& =I\left(\tilde{V}^{N}\left[\left(\mathcal{V}_{U \mid Y} \backslash \mathcal{V}_{U \mid \tilde{X}}\right) \cup \mathcal{F} \cup \mathcal{I}_{J}\right](W) ; X^{N}(W) \mid \mathcal{C}=C\right) \\
& =I\left(\tilde{V}^{N}\left[\left(\mathcal{V}_{U \mid Y} \backslash \mathcal{V}_{U \mid X}\right) \cup\left(\mathcal{V}_{U \mid X} \backslash \mathcal{V}_{U \mid \tilde{X}}\right) \cup \mathcal{F} \cup \mathcal{I}_{J}\right](W) ;\right. \\
& \left.\quad X^{N}(W) \mid \mathcal{C}=C\right) \\
& =I\left(\tilde{V}^{N}\left[\left(\mathcal{V}_{U \mid Y} \backslash \mathcal{V}_{U \mid X}\right) \cup \mathcal{F} \cup \mathcal{I}_{J}\right](W) ; X^{N}(W) \mid\right. \\
& \left.\quad \tilde{V}^{N}\left[\mathcal{V}_{U \mid X} \backslash \mathcal{V}_{U \mid \tilde{X}}\right](W), \mathcal{C}=C\right) \\
& \quad+I\left(\tilde{V}^{N}\left[\mathcal{V}_{U \mid X} \backslash \mathcal{V}_{U \mid \tilde{X}}\right](W) ; X^{N}(W) \mid \mathcal{C}=C\right) \\
& \stackrel{(b)}{\leq} \quad H\left(\tilde{V}^{N}\left[\left(\mathcal{V}_{U \mid Y} \backslash \mathcal{V}_{U \mid X}\right) \cup \mathcal{F} \cup \mathcal{I}_{J}\right](W) \mid \mathcal{C}=C\right)+\delta \\
& \leq\left|\left(\mathcal{V}_{U \mid Y} \backslash \mathcal{V}_{U \mid X}\right) \cup \mathcal{F} \cup \mathcal{I}_{J}\right|+\delta \\
& =\left|\mathcal{V}_{U \mid Y}\right|-\left|\mathcal{V}_{U \mid X}\right|+|\mathcal{F}|+\left|\mathcal{I}_{J}\right|+\delta
\end{aligned}
$$

where $\delta \rightarrow 0$ as $N \rightarrow \infty$; (a) follows from the fact that the biometric sequence $X^{N}(W)$ of user $W$ is independent of the helper data of other users; (b) follows from Lemma 5.

Combining (18), (23), (24), and (48), we have that

$$
\begin{aligned}
& \frac{I\left(\{J(i)\}_{i=1}^{M_{I}} ; X^{N}(W) \mid \mathcal{C}=C\right)}{N} \\
& \quad \stackrel{N \rightarrow \infty}{\longrightarrow} I(U ; X)-I(U ; Y)+R_{I}+2 \epsilon,
\end{aligned}
$$

where we used $U-X-Y$.

Combining (25), (27), (38), (45), (46), and (49), we complete the proof of Theorem 2 . 


\section{REFERENCES}

[1] J. L. Wayman, A. K. Jain, D. Maltoni, and D. Maio, Biometric systems: Technology, design and performance evaluation. Springer Science \& Business Media, 2005.

[2] A. K. Jain, A. Ross, and S. Pankanti, "Biometrics: a tool for information security," IEEE Trans. Inf. Forensics Security, vol. 1, no. 2, pp. 125-143, 2006.

[3] F. Willems, T. Kalker, J. Goseling, and J. P. Linnartz, "On the capacity of a biometrical identification system," in Proc. IEEE Int. Symp. Inf. Theory (ISIT). IEEE, Jun. 2003, pp. 82-87.

[4] E. Tuncel, "Capacity/storage tradeoff in high-dimensional identification systems," IEEE Trans. Inf. Theory, vol. 55, no. 5, pp. 2097-2106, 2009.

[5] F. Farhadzadeh and F. M. J. Willems, "Identification rate, search and memory complexity tradeoff: Fundamental limits," IEEE Trans. Inf. Theory, vol. 62, no. 11, pp. 6173-6188, Nov. 2016.

[6] M. T. Vu, T. J. Oechtering, and M. Skoglund, "Hierarchical identification with pre-processing," IEEE Trans. Inf. Theory, vol. 66, no. 1, pp. 82$113,2019$.

[7] L. Zhou, M. T. Vu, T. J. Oechtering, and M. Skoglund, "Two-stage biometric identification systems without privacy leakage," IEEE J. Sel. Areas Inf. Theory, 2021.

[8] T. Ignatenko and F. M. J. Willems, "Biometric systems: Privacy and secrecy aspects," IEEE Trans. Inf. Forensics Security, vol. 4, no. 4, pp. 956-973, Dec. 2009.

[9] _ - "Fundamental limits for privacy-preserving biometric identification systems that support authentication," IEEE Trans. Inf. Theory, vol. 61, no. 10 , pp. 5583-5594, Oct 2015.

[10] K. Kittichokechai and G. Caire, "Secret key-based identification and authentication with a privacy constraint," IEEE Trans. Inf. Theory, vol. 62, no. 11, pp. 6189-6203, 2016.

[11] V. Yachongka and H. Yagi, "A new characterization of the capacity region of identification systems under noisy enrollment," in 54th Annu. Conf. Inf. Sciences Systems. IEEE, 2020, pp. 1-6.

[12] L. Zhou, M. T. Vu, T. J. Oechtering, and M. Skoglund, "Fundamental limits for biometric identification systems without privacy leakage," in 2019 57th Annu. Allerton Conf. Commun., Control, Comput. IEEE, 2019, pp. 1105-1112.

[13] O. Günlü and G. Kramer, "Privacy, secrecy, and storage with multiple noisy measurements of identifiers," IEEE Trans. Inf. Forensics Security, vol. 13 , no. 11, pp. 2872-2883, 2018.

[14] M. T. Vu, "Perspectives on identification systems," Ph.D. dissertation, KTH Royal Institute of Technology, 2019.

[15] E. Arikan, "Source polarization," in 2010 IEEE Int. Symp. Inf. Theory. IEEE, 2010, pp. 899-903.

[16] R. A. Chou, M. R. Bloch, and E. Abbe, "Polar coding for secret-key generation," IEEE Trans. Inf. Theory, vol. 61, no. 11, pp. 6213-6237, 2015.

[17] L. Zhou, T. J. Oechtering, and M. Skoglund, "Fundamental limits-achieving polar code designs for biometric identification and authentication," manuscript in preparation. [Online]. Available: https://people.kth.se/\%7Elinghui/manuscript.pdf

[18] I. Csiszár and J. Körner, Information theory: coding theorems for discrete memoryless systems. Cambridge University Press, 2011. 